\title{
ATOMISTIC SIMULATION OF EJECTA PRODUCTION FROM NANOCRYSTALLINE CU WITH A GROOVED SURFACE UNDER SHOCKWAVE LOADING
}

\author{
AN-MIN HE \\ Institute of Applied Physics and Computational Mathematics, Beijing, China
}

\begin{abstract}
Large-scale molecular dynamics simulations are carried out to investigate the ejecta production from nanocrystalline $\mathrm{Cu}$ with a grooved surface under various shock intensities. The microjetting processes from the sample before and after shock-reduced melting are obtained, and the effects of polycrystalline as well as solid-liquid transition on ejecta properties are analyzed. It is found that polycrystalline structure has a significant influence on microjetting properties, including the critical condition of microjet formation and the total mass of ejecta, before shock-induced melting. After shock-induced melting, the microjetting from the nanocrystalline system tends to be consistent with the monocrystalline system.

Keywords: ejecta, molecular dynamics, nanocrystal.
\end{abstract}

\section{INTRODUCTION}

Periodic micron-scale surface perturbations are usually generated on the surface of metal during machining. When a shock wave generated by explosive or high energy laser reflects from the surface, microjet usually forms, which expands continuously and finally fragments into a great number of small particles called ejecta [1]. Due to its great importance in many fields such as inertial confinement fusion (ICF) and implosion dynamics, extensively experimental and theoretical studies have been conducted on this phenomenon [2]-[4]. Although a great progress in theoretical modelling of ejecta is achieved in recent years [5], [6], some underlying mechanisms related to microjetting process still remain unrevealed.

In the area of ejecta research, molecular dynamics (MD) simulation, which has been proven a powerful tool to explore the dynamic responses of material under extreme conditions, has been widely adopted to study the microscopic mechanism of ejecta production. However, previous works mainly focus on single crystals [7], and ejecta production from a more realistic polycrystalline remains unexplored. Here, we perform largescale MD simulations to investigate the ejecta production from nanocrystalline $\mathrm{Cu}$ with a grooved surface under various shock loading conditions.

\section{MODEL AND SIMULATION METHODS}

Classical MD simulations are performed to study ejecta production from nanocrystalline $\mathrm{Cu}$. The initial specimen is an idealized hexagonal columnar nanocrystal with a wedged surface groove as shown in Fig. 1. The atomic configuration of the columnar nanocrystal, which is composed of three types of idealized hexagonal columnar grains, is the same as the one in our previous work [8]. Type 1 grain is the reference grain with [100], [010], and [001] crystallographic directions along the $\mathrm{x}-, \mathrm{y}-$, and $\mathrm{z}$-axes. Type 2 and 3 grains are rotated around the columnar axis by $60^{\circ}$ and $30^{\circ}$ relative to type 1 grain, respectively. The depth and vertex angle of the groove are $10 \mathrm{~nm}$ and $120^{\circ}$, respectively. For comparison, a monocrystalline $\mathrm{Cu}$ with the same surface perturbation is also constructed. The widely used Embedded Atom Model (EAM) potential developed by Mishin et al. [9] is adopted. Prior to shock wave loading, the crystal is equilibrated at $300 \mathrm{~K}$ and zero pressure with the constant-pressure-temperature 
(NPT) ensemble and three-dimensional (3D) periodic boundary conditions. Then a shock wave is generated using the momentum mirror method, where the sample impacts a wall of infinite mass at a given velocity of $-u_{p}$ along the $\mathrm{x}$-axis, and a shock wave is induced in the opposite direction. The shock intensity can be varied by changing $u_{p}$.

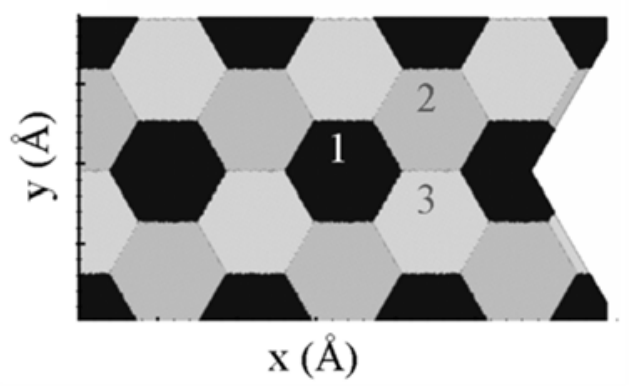

Figure 1: Initial configuration of the nanocrystalline $\mathrm{Cu}$ with a grooved surface.

\section{RESULTS AND DISCUSSION}

The microjetting processes of the nano- and monocrystalline $\mathrm{Cu}$ under different shock intensities are studied. Fig. 2 shows representative pictures of microjet formation for $u_{p}=$ $1.5 \mathrm{~km} / \mathrm{s}$, where the specimens remain solid during shock compression and subsequent unloading. For the monocrystal (Fig. 2(b)), it can be seen that a typical microjet forms due to the interaction of shock wave and surface groove, which expands under velocity gradient and finally breaks up into ejecta particles. However, for the case of nanocrystal (Fig. 2(a)), although a microjet is produced at the earlier times ( $40 \mathrm{ps})$, it stops expanding and shrinks to the metal surface. This phenomenon is attributed to the different shockwave profiles in the two samples. A shockwave with a ramp front travels inside the nanocrystal. When it reaches the surface and interacts with the groove, a microjet with lower tip velocity compared to the monocrystal case forms. As a result, the kinetic energy of the microjet is not big enough to overcome the effect of surface tension and the microjet finally becomes a liquid surface bump.

(a)

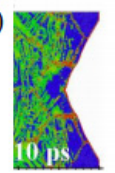

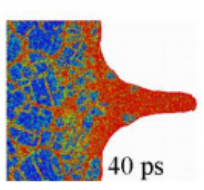

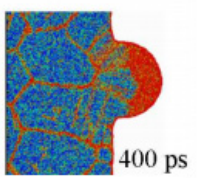

(b)

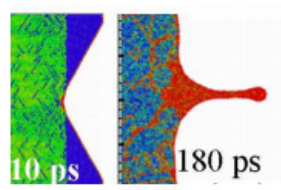

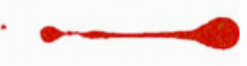

Figure 2: Morphology evolution of microjet for (a) nano- and (b) monocrystalline $\mathrm{Cu}$ shocked at $u_{p}=1.5 \mathrm{~km} / \mathrm{s}$.

As the shock intensity increases to $u_{p}=2.0 \mathrm{~km} / \mathrm{s}$ shown in Fig. 3, partial melting in the nanocrystal occurs during shock unloading, resulting in a solid-liquid mixing state in the bulk (20 ps in Fig. 3(a)). However, for the monocrystal, only a limited surface region transforms into liquid state (20 ps in Fig. 3(b)). As a consequence, the total mass of microjet from the nanocrystal is higher than that from the monocrystal, especially in the jet root region. 
As the shock intensity increases further to $u_{p}=3.5 \mathrm{~km} / \mathrm{s}$ as shown in Fig. 4, shock induced melting takes place both in the nano- and monocrystals. Therefore, the two microjets form and grow in liquid states, resulting in approximately identical jetting states.

(a)
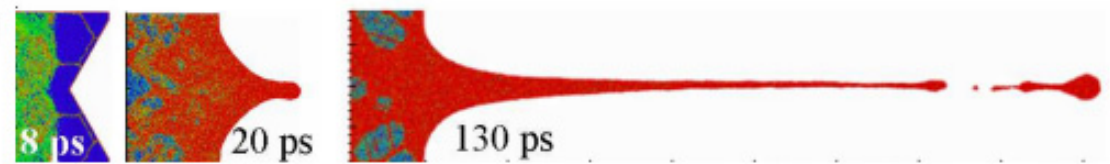

(b)
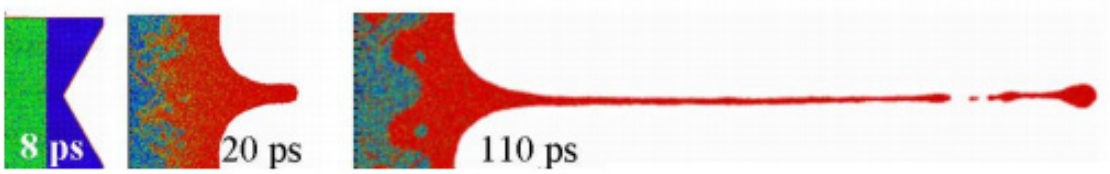

Figure 3: Morphology evolution of microjet for (a) nano- and (b) monocrystalline $\mathrm{Cu}$ shocked at $u_{p}=2.0 \mathrm{~km} / \mathrm{s}$.

(a)
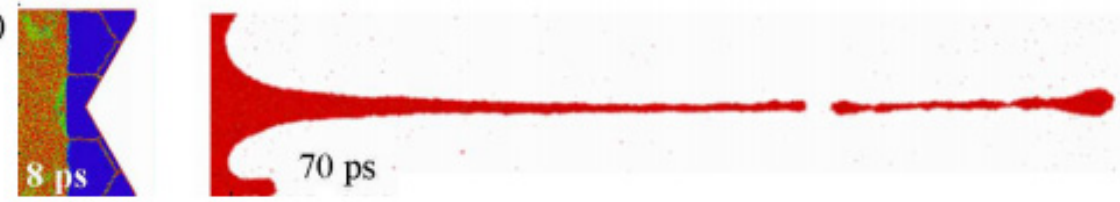

(b)
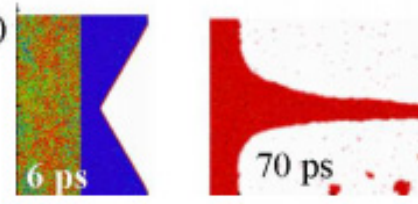

Figure 4: Morphology evolution of microjet for (a) nano- and (b) monocrystalline $\mathrm{Cu}$ shocked at $u_{p}=3.5 \mathrm{~km} / \mathrm{s}$.

The variation of jetting factor with $u_{\mathrm{p}}$ is displayed in Fig. 5. The jetting factor $R_{\mathrm{ej}}$ is defined as

$$
R_{\mathrm{ej}}=\frac{m_{\mathrm{ej}}}{\rho V_{\mathrm{def}}}
$$

where $m_{\mathrm{ej}}$ is the total mass of microjet above the free surface, $\rho$ is the material density, and $V_{\text {def }}$ is the initial volume of the surface groove. It can be clearly seen in Fig. 5 that the jetting factors are nearly identical in the shock induced melting region $\left(u_{p} \geq 3.5 \mathrm{~km} / \mathrm{s}\right)$, consistent with the jetting states given in Fig. 4. In the solid and mixing region $\left(2.0 \mathrm{~km} / \mathrm{s} \leq u_{p} \leq\right.$ $3.0 \mathrm{~km} / \mathrm{s}$ ), the jetting factor for the nanocrystal is obviously higher than that of the monocrystal due to the different melting dynamics as explained above.

The variation of microjet tip velocity with post-shock particle velocity for the two systems is presented in Fig. 6. It can be seen that the tip velocity of microjet in the nanocrystal is almost the same as in the monocrystal case, and increases linearly with post-shock particle velocity. 


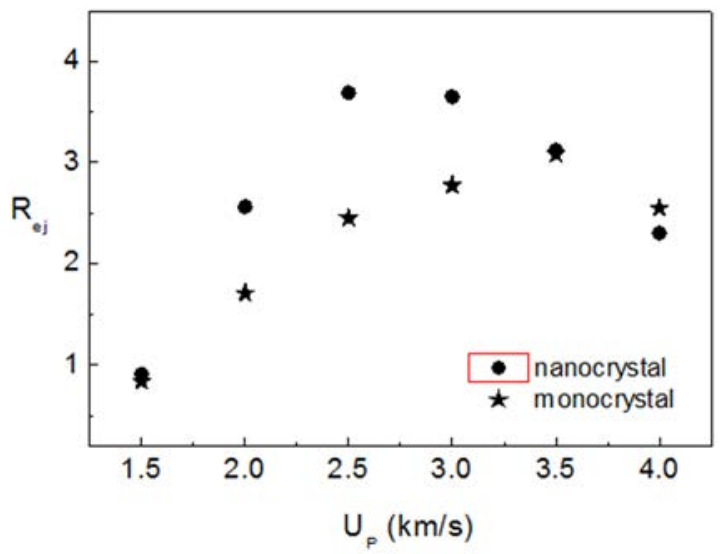

Figure 5: Variation of jetting factor with post-shock particle velocity.

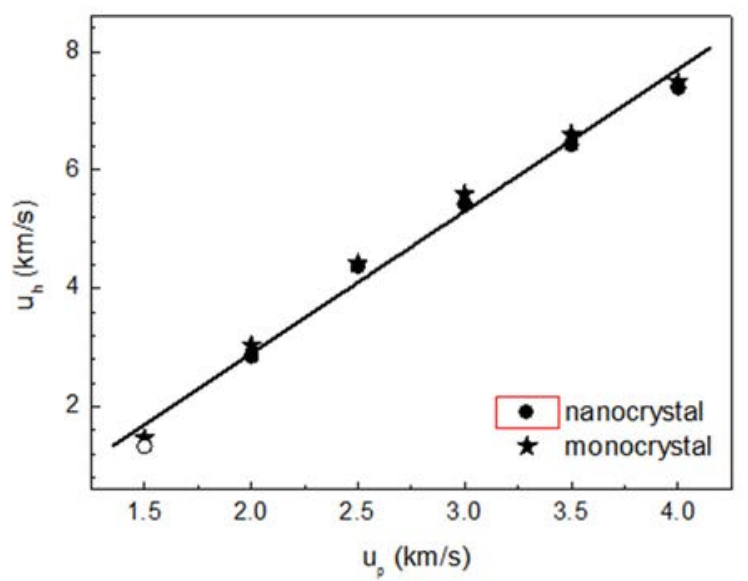

Figure 6: Variation of microjet tip velocity with post-shock particle velocity.

\section{CONCLUSION}

We performed molecular dynamics simulations to investigate the ejecta production from nanocrystal $\mathrm{Cu}$ with a grooved surface under various shock loading conditions. It is found that the microjetting morphology and the total mass of ejecta are different from the monocrystal in the shock pressure region before shock induced melting, caused by the special features of shock wave propagation and the melting dynamics in the nanocrystal. However, the tip velocity of microjet is independent on the micro-structure of the system.

\section{ACKNOWLEDGEMENTS}

This work was supported by the National Natural Science Foundation of China (Grant No. U1530261), and the Science Challenge Project (Grant Nos. TZ2016001 and TZ2018001). 


\section{REFERENCES}

[1] Asay, J.R., Mix, L.P. \& Perry, F.C., Ejection of material from shocked surfaces. Appl. Phys. Lett., 29, p. 284, 1976.

[2] Sorenson, D.S., Minich, R.W., Romero, J.L., Tunnel, T.W. \& Malone, R.M., Ejecta particle size distributions for shock loaded Sn and Al metals. J. Appl. Phys., 92, p. 5830, 2002.

[3] Buttler, W.T. et al., Dynamic comparisons of piezoelectric ejecta diagnostics. J. Appl. Phys., 101, p. 063547, 2007.

[4] Zellner, M.B. et al., Probing the underlying physics of ejecta production from shocked Sn samples. J. Appl. Phys., 103, p. 123502, 2008.

[5] Cherne, F.J., Hammerberg, J.E., Andrews, M.J., Karkhanis, V. \& Ramaprabhu, P., On shock driven jetting of liquid from non-sinusoidal surfaces into a vacuum. J. Appl. Phys., 118(18), p. 185901, 2015.

[6] He, A.-M., Liu, J., Liu, C. \& Wang, P., Numerical and theoretical investigation of jet formation in elastic-plastic solids. J. Appl. Phys., 124, p. 185902, 2018.

[7] Shao, J.L., Wang, P., He, A.M., Duan, S.Q. \& Qin, C.S., Atomistic simulations of shockinduced microjet from a grooved aluminium surface. J. Appl. Phys., 113, p. 153501, 2013.

[8] He, A.M., Duan, S.Q., Shao, J.L., Wang, P. \& Luo, S.N., Local and bulk melting of shocked columnar nanocrystalline $\mathrm{Cu}$ : Dynamics, anisotropy, premelting, superheating, supercooling, and re-crystallization. J. Chem. Phys., 139, p. 074502, 2013.

[9] Mishin, Y., Mehl, M.J., Papaconstantopoulos, D.A., Voter, A.F. \& Kress, J.D., Structural stability and lattice defects in copper: Ab initio, tight-binding, and embeddedatom calculations. Phys. Rev. B, 63(22), p. 224106, 2001. 